\title{
CESÀRO SUMMABILITY OF DOUBLE WALSH-FOURIER SERIES
}

\author{
F. MÓRICZ, F. SCHIPP AND W. R. WADE
}

\begin{abstract}
We introduce quasi-local operators (these include operators of Calderón-Zygmund type), a hybrid Hardy space $\mathbf{H}^{\sharp}$ of functions of two variables, and we obtain sufficient conditions for a quasi-local maximal operator to be of weak type $(\sharp, 1)$. As an application, we show that Cesàro means of the double Walsh-Fourier series of a function $f$ converge a.e. when $f$ belongs to $\mathbf{H}^{\sharp}$. We also obtain the dyadic analogue of a summability result of Marcienkiewicz and Zygmund valid for all $f \in L^{1}$ provided summability takes place in some positive cone.
\end{abstract}

\section{INTRODUCTION}

The problem of a.e. Cesàro summability is "quite delicate" in any local field setting (Taibleson [8, p. 114]). The dyadic case is no exception (see Fine [3]).

For double trigonometric Fourier series, Marcinkiewicz and Zygmund [4] proved that $\sigma_{m, n}(f) \rightarrow f$ a.e. as $m, n \rightarrow \infty$ provided the integral lattice points $(m, n)$ remain in some positive cone, i.e., provided $m / n \leq \beta$ and $n / m \leq \beta$ for some fixed parameter $\beta \geq 1$.

During the last decade several attempts have been made to obtain a Walsh analogue of this result. Apart from growth estimates in [9] and the almost trivial fact that double Walsh-Fourier series of $L^{p}$ functions are Cesàro summable in $L^{p}$ norm for $1 \leq p<\infty$, little is yet known. Part of the problem is that the classical Fejér kernels are dominated by decreasing functions whose integrals are bounded but this property fails to hold for the one-dimensional Walsh-Fejer kernels. This growth difference is exacerbated in higher dimensions so that the trigonometric techniques are not powerful enough for the Walsh case.

We obtain positive results concerning a.e. Cesàro summability of double Walsh-Fourier series. For $f \in L \log ^{+} L$ we show that Cesàro summability holds with no restriction on the order $(m, n)$ other than $\min \{m, n\} \rightarrow \infty$. For $f \in L^{1}$ we obtain an analogue of the Marcinkiewicz result for Cesàro means of order $\left(2^{m}, 2^{n}\right)$. Precise statements appear in $\S 3$.

In $\S 2$ we introduce quasi-local operators and show that a large class of quasilocal maximal operators are of type $\left(L^{1}, \mathbf{H}\right)$ and of weak-type $(1,1)$. Here $\mathbf{H}$ represents a dyadic Hardy space on $[0,1)$. These estimates are iterated

Received by the editors July 15, 1988 and, in revised form, September 25, 1989.

1980 Mathematics Subject Classification (1985 Revision). Primary 42C10, 43A75; Secondary 22C05, 42B20, 42B25.

Key words and phrases. Walsh functions, Cesàro summability, quasi-local operators, operators of Calderón-Zygmund type, dyadic Hardy spaces.

This research supported in part by a National Science Foundation grant (INT-8620153). 
to obtain similar inequalities for a class of two-dimensional operators which includes Cesàro means of double Walsh-Fourier series. The iteration is effected by introducing hybrid Hardy spaces of two-variables ("dyadic Hardy" in one variable and integrable in the other). For nonnegative functions, these hybrid Hardy spaces reduce to $L \log ^{+} L$.

\section{HARDY SPACES AND QUASI-LOCAL OPERATORS}

Let $\mathbf{P}$ denote the positive integers, $\mathbf{N}=\mathbf{P} \cup\{0\}$, and $Q=[0,1)$. For any set $E$, let $E^{2}$ denote the cartesian product $E \times E$. Thus $\mathbf{N}^{2}$ is the collection of integral lattice points in the first quadrant and $Q^{2}$ is the unit square.

Let $Q^{1}=Q$ and fix $j=1$ or 2 . Denote the $j$-dimensional Lebesgue measure of any subset $E$ of $Q^{j}$ by $|E|$. If $F$ is measurable on $Q^{j}$ and $\lambda>0$ let

$$
\{|F|>\lambda\} \equiv\left\{x \in Q^{j}:|F(x)|>\lambda\right\}
$$

Denote the $L^{p}\left(Q^{j}\right)$ norm of any function $F$ by $\|F\|_{p}$.

By a dyadic interval we mean one of the form $\left[p / 2^{m},(p+1) / 2^{m}\right)$ for some $p, m \in \mathbf{N}, 0 \leq p<2^{m}$. Given $m \in \mathbf{N}$ and $x \in[0,1)$ let $I_{m}(x)$ denote the dyadic interval of length $2^{-m}$ which contains $x$. Denote the collection of dyadic intervals by $\mathscr{I}^{1}$.

Let $\mathscr{J}^{2}$ denote the collection of dyadic squares, i.e., sets of the form

$$
I=I_{1} \times I_{2}
$$

where $I_{1}, I_{2} \in \mathscr{I}^{1}$ and $\left|I_{1}\right|=\left|I_{2}\right|$. Clearly, given $x=\left(x_{1}, x_{2}\right) \in Q^{2}$ and $m \in \mathbf{N}$ the dyadic square of area $2^{-2 m}$ containing $\left(x_{1}, x_{2}\right)$ is given by

$$
I_{m}\left(x_{1}\right) \times I_{m}\left(x_{2}\right) .
$$

We will also denote this set by $I_{m}(x)$.

The dyadic maximal function of an $f \in L^{1}\left(Q^{j}\right)$ is defined by

$$
f^{*}(x)=\sup _{m \in \mathbf{N}} \frac{1}{\left|I_{m}(x)\right|}\left|\int_{I_{m}(x)} f\right| \quad\left(x \in Q^{j}\right) .
$$

Recall that the dyadic Hardy space $\mathbf{H}\left(Q^{j}\right)$ is the collection of $f \in L^{1}\left(Q^{j}\right)$ such that

$$
\|f\|_{\mathbf{H}\left(Q^{j}\right)} \equiv\left\|f^{*}\right\|_{1}<\infty .
$$

In the classical proof that Calderon-Zygmund integral operators $T$ are of type $(p, p)$ for $1<p<\infty$ (see [7, p. 44], for example), a key step involves showing there is an absolute constant $C$ and an expansion factor $r$ such that if $f$ is a function of mean zero supported on a cube $I$, and if $I^{*}$ is a cube centered where $I$ is but expanded by the factor $r$, then

$$
\int_{Q^{3} \sim I^{*}} T f \leq C\|f\|_{1} .
$$

Using this as motivation, we make the following definitions. For each $I \in \mathscr{I}^{j}$ and each $r \in \mathbf{N}$ let $I^{r} \in \mathscr{I}^{j}$ be defined by $I \subseteq I^{r}$ and

$$
\left|I^{r}\right|=2^{j r}|I| \text {. }
$$


Let $L^{0}\left(Q^{j}\right)$ represent the collection of functions measurable and a.e. finite on $Q^{j}$. An operator $T: L^{1}\left(Q^{j}\right) \rightarrow L^{0}\left(Q^{j}\right)$ will be called quasi-local if there exist constants $C>0$ and $r \in \mathbf{N}$ that

$$
\int_{Q^{j} \sim I^{r}}|T f| \leq C\|f\|_{1}
$$

for all $f \in L^{1}\left(Q^{j}\right)$ and $I \in \mathscr{I}^{j}$ which satisfy

$$
\{f \neq 0\} \subseteq I
$$

and

$$
\int_{Q^{j}} f=0
$$

The following gives sufficient conditions for a maximal operator to be of type $\left(\mathbf{H}\left(Q^{j}\right), L^{1}\left(Q^{j}\right)\right)$ and of weak-type $(1,1)$.

Theorem 1. Let $\left(T_{\gamma}, \gamma \in \Gamma\right)$ be a collection of bounded sublinear operators on $L^{1}\left(Q^{j}\right)$ and set

$$
T f=\sup _{\gamma \in \Gamma}\left|T_{\gamma} f\right| \quad\left(f \in L^{1}\left(Q^{j}\right)\right) .
$$

If $T$ is quasi-local and bounded as an operator on $L^{\infty}\left(Q^{j}\right)$ then there is a constant $B>0$ (depending only on the operator norm $\|T\|_{\infty}$ and the constants of quasi-locality $C$ and $r$ ) such that

$$
\|T f\|_{1} \leq B\|f\|_{\mathbf{H}\left(Q^{j}\right)}
$$

and

$$
|\{T f>\lambda\}| \leq \frac{B}{\lambda}\|f\|_{1}
$$

for all $f \in L^{1}\left(Q^{j}\right)$ and $\lambda>0$.

Proof. A function $a \in L^{\infty}\left(Q^{j}\right)$ is called a dyadic atom if either $a=1$ or if there is an $I \in Q^{j}$ such that

$$
|a| \leq \chi_{I}|I|^{-1}
$$

and

$$
\int_{Q^{j}} a=0,
$$

where $\chi_{I}$ represents the characteristic function of $I$. Recall (see Coifman and Weiss [1]) that an $f \in L^{1}\left(Q^{j}\right)$ belongs to $\mathbf{H}\left(Q^{j}\right)$ if and only if there is a sequence of dyadic atoms $\left(a_{n}, n \in \mathbf{N}\right)$ and a sequence of numbers $\Lambda \equiv$ $\left(\lambda_{n}, n \in \mathbf{N}\right)$ such that

$$
f=\sum_{n=0}^{\infty} \lambda_{n} a_{n}
$$

and

$$
\|\Lambda\|_{l^{1}}=\sum_{n=0}^{\infty}\left|\lambda_{n}\right|<\infty .
$$


Also recall that $\Lambda$ can be chosen so that

$$
\|\Lambda\|_{l^{1}} \approx\|f\|_{\mathbf{H}\left(Q^{j}\right)} \text {. }
$$

Suppose $a$ satisfies (6) and (7) for some $I \in \mathscr{J}^{j}$. By quasi-locality and $L^{\infty}$ boundedness of $T$ we have

$$
\begin{aligned}
\int_{Q^{j}} T a & =\int_{I^{r}} T a+\int_{Q^{j} \sim I^{r}} T a \\
& \leq\|T\|_{\infty}\|a\|_{\infty}\left|I^{r}\right|+C\|a\|_{1} \\
& \leq 2^{j r}\|T\|_{\infty}+C \equiv \tilde{A} .
\end{aligned}
$$

Consequently, if $f$ has atomic decomposition (8) then

$$
\|T f\|_{1} \leq \sum_{n=0}^{\infty}\left|\lambda_{n}\right|\left\|T a_{n}\right\|_{1} \leq \widetilde{A}\|\Lambda\|_{l^{1}}
$$

for $\Lambda=\left(\lambda_{n}, n \in \mathbf{N}\right)$. Thus (4) holds for all $f \in \mathbf{H}\left(Q^{j}\right)$.

To prove (5) fix $f \in L^{1}\left(Q^{j}\right)$ and $\lambda>\|f\|_{1}$. Apply the Calderón-Zygmund decomposition (see [7], for example) to choose nonoverlapping sets $I_{n} \in \mathscr{I}^{j}$ $(n \in \mathbf{N})$ and functions $g, h \in L^{1}\left(Q^{j}\right)$ such that

$$
\begin{gathered}
f=g+h, \\
\|g\|_{\infty} \leq 4 \lambda, \\
|\Omega| \leq\|f\|_{1} / \lambda
\end{gathered}
$$

for $\Omega \equiv \bigcup_{n=0}^{\infty} I_{n}$, such that

$$
\int_{Q^{j}} h_{n}=0
$$

and

$$
\left\|h_{n}\right\|_{1} \leq 8 \lambda\left|I_{n}\right|
$$

for $h_{n}=\chi_{I_{n}} h$ and $n \in \mathbf{N}$.

Set

$$
\Omega^{r}=\bigcup_{n=0}^{\infty} I_{n}^{r}
$$

where $r \in \mathbf{N}$ is the parameter given by the quasi-locality of $T$. Notice by construction and (12) that

$$
\left|\Omega^{r}\right| \leq \frac{2^{j r}}{\lambda}\|f\|_{1}
$$

Notice also by (13) and quasi-locality that

$$
\int_{Q^{j} \sim \Omega^{r}} T h \leq \sum_{n=0}^{\infty} \int_{Q^{j} \sim I_{n}^{r}} T h_{n} \leq C \sum_{n=0}^{\infty}\left\|h_{n}\right\|_{1} .
$$

Therefore, it follows from (10), (11), (15), and (14) that

$$
\begin{aligned}
\mid\{T f & >(1+A) \lambda\}|\leq|\{T h>\lambda\} \mid \\
& \leq \frac{2^{j r}}{\lambda}\|f\|_{1}+\frac{1}{\lambda} \int_{Q^{j} \sim \Omega^{r}} T h \leq \frac{\|f\|_{1}}{\lambda}\left(2^{j r}+8 C\right)
\end{aligned}
$$


for $A=4\|T\|_{\infty}$. Since this inequality also holds for $\lambda \leq\|f\|_{1}$ we conclude by a change of variables that (5) holds and the proof of Theorem 1 is complete.

Define a hybrid dyadic maximal function for each $f \in L^{1}\left(Q^{2}\right)$ by

$$
f^{\sharp}(x, y)=\sup _{m \in \mathbf{N}} \frac{1}{\left|I_{m}(y)\right|}\left|\int_{I_{m}(y)} f(x, t) d t\right|
$$

for $(x, y) \in Q^{2}$. A function $f \in L^{1}\left(Q^{2}\right)$ is said to belong to $\mathbf{H}^{\sharp}\left(Q^{2}\right)$ if

$$
\|f\|_{\sharp} \equiv\left\|f^{\sharp}\right\|_{1}<\infty \text {. }
$$

Notice that $\mathbf{H}\left(Q^{2}\right) \subset \mathbf{H}^{\sharp}\left(Q^{2}\right) \subset L^{1}\left(Q^{2}\right)$. Also notice that if $L \log ^{+} L\left(Q^{2}\right)$ represents the collection of $f \in L^{1}\left(Q^{2}\right)$ which satisfy

$$
\int_{Q^{2}}|f| \log ^{+}|f|<\infty
$$

then $f \in L \log ^{+} L\left(Q^{2}\right)$ if and only if $|f| \in \mathbf{H}^{\sharp}\left(Q^{2}\right)$.

Given functions $V_{0}, V_{1}$ defined on $Q$ define the Kronecker product of $V_{0}$ and $V_{1}$ on $Q^{1}$ by

$$
\left(V_{0} \times V_{1}\right)(x, y)=V_{0}(x) V_{1}(y) .
$$

Let $*$ denote the dyadic convolution on $L^{1}\left(Q^{j}\right)$, for example,

$$
(g * h)(t)=\int_{Q} g(t+s) h(s) d s
$$

for $g, h \in L^{1}(Q)$ and $t \in Q$ (see Fine [2]). The following result shows that a Kronecker product of a maximal convolution operator of weak-type $(1,1)$ with one of type $\left(\mathbf{H}(Q), L^{1}(Q)\right)$ is an operator of weak type $(\sharp, 1)$ on $Q^{2}$ provided one of the kernels is nonnegative.

Theorem 2. Let $\left(V_{n}^{i}, n \in \mathbf{N}\right), i=0,1$, be sequences of $L^{1}(Q)$ functions. Define one-dimensional operators

$$
T^{i} h \equiv \sup _{m \in \mathbf{N}}\left|h * V_{m}^{i}\right|
$$

for $h \in L^{1}(Q), i=0,1$, and suppose there exist absolute constants $A_{0}, A_{1}$ such that

$$
\left|\left\{T^{0} h>\lambda\right\}\right| \leq \frac{A_{0}}{\lambda}\|h\|_{1}
$$

and

$$
\left\|T^{1} h\right\|_{1} \leq A_{1}\|h\|_{\mathbf{H}(Q)}
$$

for all $h \in L^{1}(Q)$ and $\lambda>0$. If $V_{m}^{0} \geq 0$ for all $m \in \mathbf{N}$ and

$$
T f \equiv \sup _{\left(n_{1}, n_{2}\right) \in \mathbf{N}^{2}}\left|f *\left(V_{n_{1}}^{0} \times V_{n_{2}}^{1}\right)\right|
$$

then

$$
|\{T f>\lambda\}| \leq \frac{A_{0} A_{1}}{\lambda}\|f\|_{\sharp}
$$

for all $f \in \mathbf{H}^{\sharp}\left(Q^{2}\right)$ and $\lambda>0$. 
Proof. Fix $f \in \mathbf{H}^{\sharp}\left(Q^{2}\right)$ and $\lambda>0$. Consider the function

$$
F_{y}(u)=F(u, y) \equiv \sup _{n_{2} \in \mathbf{N}}\left|\int_{Q} f(u, v) V_{n_{2}}^{1}(y+v) d v\right|
$$

defined for all $u, y \in Q$. Since Lebesgue measure is translation invariant with respect to dyadic addition $\dot{+}$ (see Fine [2]) and $V_{n_{1}}^{0}$ is nonnegative it is easy to check that

$$
\left|\left(f *\left(V_{n_{1}}^{0} \times V_{n_{2}}^{1}\right)\right)(x, y)\right| \leq \sup _{n_{1} \in \mathbf{N}} \int_{Q} V_{n_{1}}^{0}(x+u) F_{y}(u) d u
$$

for $n_{2} \in \mathbf{N}$. Consequently,

$$
(T f)(x, y) \leq\left(T^{0} F_{y}\right)(x)
$$

for $(x, y) \in Q^{2}$.

Let $m(y)$ be the (one-dimensional) Lebesgue measure of $\{x \in Q:(T f)(x, y)$ $>\lambda\}$. Notice by (16) and (20) that

$$
m(y) \leq\left|\left\{T^{0} F_{y}>\lambda\right\}\right| \leq \frac{A_{0}}{\lambda}\left\|F_{y}\right\|_{1} .
$$

Also notice by (17) and (19) that

$$
\int_{Q} F(u, y) d y \leq A_{1} \int_{Q} f^{\sharp}(u, v) d v
$$

for all $u \in Q$. Therefore,

$$
|\{T f>\lambda\}|=\int_{Q} m(y) d y \leq \frac{A_{0}}{\lambda} \int_{Q} \int_{Q} F(u, y) d u d y \leq \frac{A_{0} A_{1}}{\lambda}\left\|f^{\sharp}\right\|_{1}
$$

and the proof of Theorem 2 is complete.

\section{CesÀro summability of double Walsh-Fourier Series}

Let $\left(w_{n}, n \in \mathbf{N}\right)$ represent the one-dimensional Walsh-Paley system, recall that

$$
w_{n}(t) w_{n}(s)=w_{n}(t+s) \quad(s, t \in Q, n \in \mathbf{N})
$$

and that the Walsh-Dirichlet kernels

$$
D_{n} \equiv \sum_{k=0}^{n-1} w_{k} \quad(n \in \mathbf{P})
$$

satisfy

$$
D_{2^{m}}(t)= \begin{cases}2^{m}, & t \in\left[0,2^{-m}\right), \\ 0, & t \in\left[2^{-m}, 1\right),\end{cases}
$$

for $m \in \mathbf{N}$ (see Fine [2]). Also recall that the Walsh-Fejér kernels

$$
K_{m}=\frac{1}{m} \sum_{n=1}^{m} D_{n} \quad(m \in \mathbf{P})
$$


satisfy

$$
\left|K_{m}(t)\right| \leq \sum_{j=0}^{n-1} 2^{j-n} \sum_{i=j}^{n-1}\left(D_{2^{i}}(t)+D_{2^{i}}\left(t+2^{-j-1}\right)\right)
$$

for $t \in Q, n, m \in \mathbf{P}, 2^{n-1} \leq m<2^{n}$ and

$$
K_{2^{n}}(t)=\frac{1}{2}\left(2^{-n} D_{2^{n}}(t)+\sum_{j=0}^{n} 2^{j-n} D_{2^{n}}\left(t+2^{-j-1}\right)\right)
$$

for $t \in Q, n \in \mathbf{N}$ (see [6]). Notice by changing the order of summation in (22) that (23) implies

$$
\begin{aligned}
\left.\mid K_{m}(t)\right) \mid & \leq 2^{-n} \sum_{i=0}^{n-1} \sum_{j=0}^{i} 2^{j}\left(D_{2^{i}}\left(t+2^{-j-1}\right)+D_{2^{i}}(t)\right) \\
& =2^{-n} \sum_{i=0}^{n-1}\left(2^{i+1} K_{2^{i}}(t)+\left(2^{i+1}-2\right) D_{2^{i}}(t)\right) \\
& \leq 2^{-n} \sum_{i=0}^{n-1} 2^{i+1}\left(K_{2^{i}}(t)+D_{2^{i}}(t)\right)
\end{aligned}
$$

for $t \in Q, n, m \in \mathbf{P}$, and $2^{n-1} \leq m<2^{n}$. Since $D_{2^{i}} \leq 2 K_{2^{i}}$ for $i \in \mathbf{N}$ it follows that

$$
\left|K_{m}\right| \leq \frac{6}{2^{n}} \sum_{i=0}^{n-1} 2^{i} K_{2^{i}}
$$

for $n, m \in \mathbf{P}, 2^{n-1} \leq m<2^{n}$.

For $m=\left(m_{1}, m_{2}\right) \in \mathbf{N}^{2}$ and $f \in L^{1}\left(Q^{2}\right)$ the Cesàro mean of order $m$ of the double Walsh-Paley-Fourier series of $f$ is given by

$$
\sigma_{m} f=f *\left(K_{m_{1}} \times K_{m_{2}}\right) \text {. }
$$

We shall examine the maximal functions

$$
\sigma^{*} f=\sup _{m \in \mathbf{P}^{2}}\left|\sigma_{m} f\right|, \quad \sigma f=\sup _{\left(n_{1}, n_{2}\right) \in \mathbf{N}^{2}}\left|\sigma_{\left(2^{n_{1}}, 2^{n_{2}}\right)} f\right|
$$

and

$$
\sigma_{\alpha} f=\sup _{\left|n_{1}-n_{2}\right| \leq \alpha}\left|\sigma_{\left(2^{n_{1}}, 2^{n_{2}}\right)} f\right|
$$

for $\alpha \geq 0$.

In the next section we prove

Theorem 3. There is an absolute constant $A>0$ such that

$$
|\{\sigma f>\lambda\}| \leq \frac{A}{\lambda}\|f\|_{\sharp}
$$

and

$$
\left|\left\{\sigma^{*} f>\lambda\right\}\right| \leq \frac{36 A}{\lambda}\||f|\|_{\sharp}
$$

for every $f \in \mathbf{H}^{\sharp}\left(Q^{2}\right)$ and $\lambda>0$. 
It is easy to see that the two-dimensional Walsh polynomials are dense in $\mathbf{H}^{\sharp}\left(Q^{2}\right)$, for by $(21)$ the dyadic square partial sums of the double Walsh-PaleyFourier series of an $f \in L^{1}\left(Q^{2}\right)$ satisfy

$$
\begin{aligned}
\left(S_{\left(2^{m}, 2^{m}\right)} f\right)(u, v) & \equiv\left(f *\left(D_{2^{m}} \times D_{2^{m}}\right)\right)(u, v) \\
& =\frac{1}{\left|I_{m}(u, v)\right|} \int_{I_{m}(u, v)} f
\end{aligned}
$$

for any $m \in \mathbf{N}$ and $(u, v) \in Q^{2}$. Consequently, Theorem 3 and the usual density argument (see Marcinkiewicz and Zygmund [4]) imply

Corollary 1. If $|f| \in \mathbf{H}^{\sharp}\left(Q^{2}\right)$ (in particular, if $f \in L \log ^{+} L\left(Q^{2}\right)$ ) then

$$
\sigma_{\left(n_{1}, n_{2}\right)} f \rightarrow f \text { a.e. }
$$

as $\min \left\{n_{1}, n_{2}\right\} \rightarrow \infty$.

Concerning the operators $\sigma_{\alpha}$ we will prove

Theorem 4. For each $\alpha \geq 0$ there is an absolute constant $B_{\alpha}>0$ such that

$$
\left\|\sigma_{\alpha} f\right\|_{1} \leq B_{\alpha}\|f\|_{\mathbf{H}\left(Q^{2}\right)}
$$

and

$$
\left|\sigma_{\alpha} f>\lambda\right| \leq \frac{B_{\alpha}}{\lambda}\|f\|_{1}
$$

for every $f \in L^{1}\left(Q^{2}\right)$ and $\lambda>0$.

Hence we get

Corollary 2. If $f \in L^{1}\left(Q^{2}\right)$ and $\alpha \geq 0$ then

$$
\sigma_{\left(2^{n_{1}}, 2^{n_{2}}\right)} f \rightarrow f \quad \text { a.e. }
$$

as $\min \left\{n_{1}, n_{2}\right\} \rightarrow \infty$ and $\left|n_{1}-n_{2}\right| \leq \alpha$.

In particular, the Walsh analogue of the Marcinkiewicz-Zygmund result holds for Cesàro means of dyadic orders. The original problem (see [9]) of whether

$$
\sigma_{\left(n_{1}, n_{2}\right)} f \rightarrow f \quad \text { a.e. }
$$

as $\min \left\{n_{1}, n_{2}\right\} \rightarrow \infty$ and $2^{-\alpha} \leq n_{1} / n_{2} \leq 2^{\alpha}$ is still open.

\section{Proofs of Theorem 3 AND 4}

By (24)

$$
\sigma^{*} f \leq 36 \sigma(|f|) \text {. }
$$

By Theorem 1 for $j=1$ and Theorem 2, the proof of Theorem 3 will be complete if we show

$$
T^{0} h \equiv \sup _{m \in \mathbf{N}}\left|h * K_{2^{m}}\right| \quad\left(h \in L^{1}(Q)\right)
$$

is bounded on $L^{\infty}(Q)$ and quasi-local. 
Boundedness is easy, since $\left\|K_{2^{m}}\right\|_{1}=1$ (see (21) and (23)). To show $T^{0}$ is quasi-local (with $r=0$ ) fix $h \in L^{1}(Q)$ with

$$
\int_{Q} h=0
$$

and

$$
\{h \neq 0\} \subseteq I
$$

for some $I \in \mathscr{I}^{1}$. Choose $l \in \mathbf{N}$ such that $|I|=2^{-l}$. Fix $0 \leq j \leq n$ and for each $x \in Q$ define

$$
\tau(j, x) D_{2^{n}}(t)=D_{2^{n}}\left(t+x+2^{-j-1}\right) \quad(t \in Q) .
$$

By (21) the function $\tau(j, x) D_{2^{n}}$ is supported on a dyadic interval of length $2^{-n}$. Consequently, for any $x \in Q$ if $n \leq l$ then we have by (30) and (31) that

$$
\int_{Q} h \cdot \tau(j, x) D_{2^{n}}=0 .
$$

Also, if $n>l$ but $l \leq j$ and $x \notin I$ then we have by (31) that

$$
h \cdot \tau(j, x) D_{2^{n}}=0 \text {. }
$$

Therefore, it follows from (23) that

$$
\begin{aligned}
\left|\left(h * K_{2^{n}}\right)(x)\right| & \leq \sum_{j=0}^{l-1} 2^{j-n} \int_{Q}|h| \tau(j, x) D_{2^{n}} \\
& \leq \sum_{j=0}^{l-1} 2^{j-l} \int_{Q}|h| \tau(j, x) D_{2^{l}}
\end{aligned}
$$

for all $x \in Q \sim I$. Since this last sum is independent of $n$ we conclude that

$$
\int_{Q \sim I}\left(T^{0} h\right)(x) d x \leq \sum_{j=0}^{l-1} 2^{j-l}\|h\|_{1} \leq\|h\|_{1}
$$

i.e., $T^{0}$ is quasi-local.

To prove Theorem 4 we apply Theorem 1 to $\sigma_{\alpha}$ for $j=2$. Since $\sigma_{\alpha} f \leq \sigma f$ it is clear that $\sigma_{\alpha}$ is bounded on $L^{\infty}\left(Q^{2}\right)$. To show $\sigma_{\alpha}$ is quasi-local choose $r \in \mathbf{N}$ such that $r-1<\alpha \leq r$. Let $f \in L^{1}\left(Q^{2}\right)$ satisfy

$$
\int_{Q^{2}} f=0
$$

and

$$
\{f \neq 0\} \subseteq I
$$

from some $I \in \mathscr{I}^{2}$. Choose $l \in \mathbf{N}$ such that $|I|=2^{-2 l}$ and proceed as we did for $j=1$.

Namely, fix $n=\left(n_{1}, n_{2}\right) \in \mathbf{N}^{2}, 0 \leq j_{1} \leq n_{1}, 0 \leq j_{2} \leq n_{2}$, and notice by (21), (32) and (33) that

$$
\int_{Q} f \cdot \tau\left(j_{1}, x_{1}\right) D_{2^{n_{1}}} \cdot \tau\left(j_{2}, x_{2}\right) D_{2^{n_{2}}}=0
$$


for $\left(x_{1}, x_{2}\right) \in Q^{2}, n_{1} \leq l$ and $n_{2} \leq l$. On the other hand, if $n_{1}>l$ or $n_{2}>l$ then the condition $\left|n_{1}-n_{2}\right| \leq \alpha$ implies

$$
n_{1}, n_{2} \geq l-\alpha \geq l-r .
$$

Let $j_{1} \geq l-r$ or $j_{2} \geq l-r$. If (34) holds and if $x=\left(x_{1}, x_{2}\right) \in Q^{2} \sim I^{r}$ then by (21) and (33) we have

$$
f \cdot \tau\left(j_{1}, x_{2}\right) D_{2^{n_{1}}} \cdot \tau\left(j_{2}, x_{2}\right) D_{2^{n_{2}}}=0 .
$$

Hence it follows from (23) that

$$
\begin{aligned}
& \mid(f *\left(K_{2^{n_{1}}} \times K_{\left.\left.2^{n_{2}}\right)\right)(x) \mid}\right. \\
& \quad \leq \sum_{j_{1}, j_{2}=0}^{l-r-1} 2^{j_{1}+j_{2}-\left(n_{1}+n_{2}\right)} \int_{Q^{2}}|f| \cdot \tau\left(j_{1}, x_{1}\right) D_{2^{n_{1}}} \cdot \tau\left(j_{2}, x_{2}\right) D_{2^{n_{2}}}
\end{aligned}
$$

for $x=\left(x_{2}, x_{2}\right) \in Q^{2} \sim I^{r}$ and $\left(n_{1}, n_{2}\right) \in \mathbf{N}^{2}$ satisfying $\left|n_{1}-n_{2}\right| \leq \alpha$. Therefore,

$$
\left(\sigma_{\alpha} f\right)(x) \leq G(x) \quad\left(x \in Q^{2} \sim I^{r}\right)
$$

where

$$
G(x) \equiv \sum_{j_{1}, j_{2}=0}^{l-r-1} 2^{j_{1}+j_{2}-2(l-r)} \int_{Q^{2}}|f| \cdot \tau\left(j_{1}, x_{1}\right) D_{2^{l-r}} \cdot \tau\left(j_{2}, x_{2}\right) D_{2^{l-r}} .
$$

But

by (21). Consequently, (35) implies

$$
\|G\|_{1} \leq\|f\|_{1}
$$

$$
\int_{Q^{2} \sim I^{r}} \sigma_{\alpha} f \leq\|f\|_{1}
$$

and we conclude that $\sigma_{\alpha}$ is quasi-local.

\section{REFERENCES}

1. R. R. Coifman and G. Weiss, Extension of Hardy spaces and their use in analysis, Bull. Amer. Math. Soc. 83 (1977), 569-645.

2. N. J. Fine, On the Walsh functions, Trans. Amer. Math. Soc. 65 (1949), 372-414.

3. __ Cesàro summability of Walsh-Fourier series, Proc. Nat. Acad. Sci. U.S.A. 41 (1955), 558-591.

4. J. Marcinkiewicz and A. Zygmund, On the summability of double Fourier series, Fund. Math. 32 (1939), 122-132.

5. F. Móricz and F. Schipp, On the integrability and $L^{1}$-convergence of double Walsh series, Acta Math. Hungar. 57 (1991), 371-380.

6. F. Schipp, Über gewissen Maximaloperatoren, Ann. Univ. Sci. Budapest Sect. Math. 18 (1975), 189-195.

7. E. M. Stein, Singular integrals and differentiability properties of functions, Princeton Univ. Press, Princeton, N. J., 1970.

8. M. H. Taibleson, Fourier analysis on local fields, Princeton Univ. Press, Princeton, N. J., 1975.

9. W. R. Wade, A growth estimate for Cesàro partial sums of multiple Walsh-Fourier series, Colloq. Math. Soc. J. Bolyai 49, Alfred Haar Memorial Conference, (Budapest, 1985), 975-991.

Department of Mathematics, University of Tennessee, Knoxville, Tennessee 37916 